\title{
Management of penile cancer
}

Penile cancer remains a relatively infrequent malignancy in North America, Europe, and Asia nevertheless it is a devastating disease which portends an adverse prognosis in those presenting with advanced disease. Similarly, the clinical and surgical care remains highly diverse across many parts of the world often applying non-evidence based approaches in their management.

The present special issue of the Translational Andrology and Urology (TAU) journal has been devised for this specific reason of providing the most up-to-date and scientifically sound diagnostic and treatment approaches to the care of patients suffering from penile cancer. We share some of the triumphant discoveries made in recent years including our better understanding of the pathophysiology and molecular pathways implicated in penile cancer particularly those involving the human papillomavirus. A significant component of this issue is dedicated to discussing the spectrum of penile sparing treatment approaches available to primary penile neoplasms. Management of the inguinal nodes which constitute the first echelon of nodes affected by tumor dissemination of penile tumors has been traditionally approached by open inguinal lymph node dissection associated with high morbidity and in consequence has been an often unpopular treatment choice. In recent years however technical advances have been made in our surgical assessment of the inguinal nodes including the adoption of minimally invasive surgery using robotics making it a more palatable option for many patients and/or treating physicians. Penile cancer patients presenting with locally advanced (cN2/3) are often best served with a multimodal approach of neoadjuvant systemic chemotherapy followed by surgery, with the data supporting such an approach highlighted. Similarly, a number of novel systemic agents and immune modulatory therapies have the potential to redefine our current treatment paradigm which will be detailed. Lastly, a topic often not given much attention is the psychosocial impact of penile cancer and its imparting treatments on patients and their families. This important topic will be discussed in the current issue.

We are confident that readers of this issue will gain the necessary knowledge and insight to improve the care of their penile cancer patients from across the world. Although we have made some significant advances, the fight against cancer rages on and we can never accept that what we have achieved is good enough until cure is achieved in all those plagued with this horrific disease.

\section{Acknowledgements}

None.

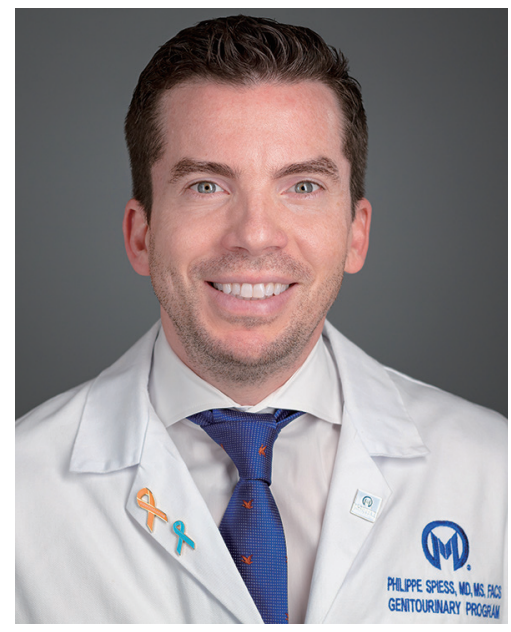

Philippe E. Spiess, Special Guest Editor

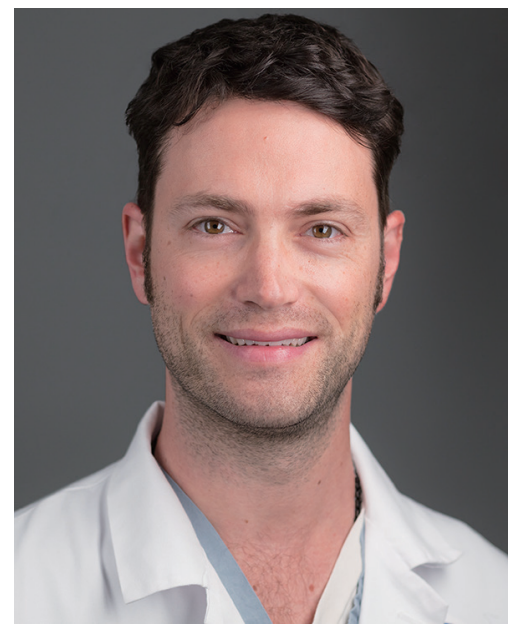

Michael A. Poch, Special Guest Editor 


\section{Philippe E. Spiess, MD, MS, FACS}

Professor of Genitourinary Oncology and Tumor Biology, Assistant Chief of Surgery, Moffitt Cancer Center, Tampa, FL, USA.

(Email: Philippe.Spiess@moffitt.org)

Michael A. Poch, MD

Assistant Professor, Department of Genitourinary Oncology, Moffitt Cancer Center, Tampa, FL, USA.

(Email: Michael.Poch@moffitt.org)

doi: 10.21037/tau.2017.09.15

Conflicts of Interest: The authors have no conflicts of interest to declare.

View this article at: http://dx.doi.org/10.21037/tau.2017.09.15

Cite this article as: Spiess PE, Poch MA. Management of penile cancer. Transl Androl Urol 2017;6(5):783-784. doi: 10.21037/tau.2017.09.15 